I nt er el ectroni $c$ angl es of group 14, 15, and 16 at ons in thei $r$ l ow l yi ng mul ti pl et st ates

\begin{tabular}{|c|c|}
\hline 著者 & KOGA Toshi kat su \\
\hline $\begin{array}{l}\text { j our nal or } \\
\text { publ i cat i on titl e }\end{array}$ & The j our nal of chem cal physi cs \\
\hline vol une & 119 \\
\hline nunber & 14 \\
\hline page $r$ ange & 7145- 7147 \\
\hline year & $2003-10-08$ \\
\hline URL & ht t p: //hdl . handl e. net /10258/829 \\
\hline
\end{tabular}




\title{
Interelectronic angles of group 14, 15, and 16 atoms in their low-lying multiplet states
}

\author{
Toshikatsu Koga \\ Department of Applied Chemistry, Muroran Institute of Technology, Muroran, Hokkaido 050-8585, Japan
}

(Received 16 June 2003; accepted 15 July 2003)

\begin{abstract}
The average interelectronic angles $\left\langle\theta_{12}\right\rangle$ in position space and $\left\langle\bar{\theta}_{12}\right\rangle$ in momentum space are examined for the multiplet states arising from the ground electronic configurations of the group 14, 15 , and 16 atoms. The angles $\left\langle\theta_{12}\right\rangle$ and $\left\langle\bar{\theta}_{12}\right\rangle$ are greater than 90 degrees with no exceptions and two electron vectors prefer inverse directions to the same direction on average. Common to all 15 atoms, it is found that in position space, lower multiplets have larger interelectronic angles $\left\langle\theta_{12}\right\rangle$ than higher multiplets. However, the opposite is true in momentum space and the interelectronic angles $\left\langle\bar{\theta}_{12}\right\rangle$ are smaller in lower multiplets. The differences in the angles $\Delta\left\langle\theta_{12}\right\rangle$ and $\Delta\left\langle\bar{\theta}_{12}\right\rangle$ between multiplets have good linear correlations with the relative stability $\Delta E$ of the multiplets. (C) 2003 American Institute of Physics. [DOI: 10.1063/1.1605937]
\end{abstract}

In connection to Hund's empirical rules (see, e.g., Ref. 1) for the relative stability of atomic multiplets, the characteristics of the multiplet states arising from $p^{2}, p^{3}$, and $p^{4}$ electronic configurations of group 14, 15, and 16 atoms in the periodic table were extensively studied and discussed in the literature. Good reviews and relevant references are found in Refs. 2-7. The properties examined in the literature include the electron-nucleus attraction energy $V_{n e}$ $=\langle-Z / r\rangle$, the electron-electron repulsion energy $V_{e e}$ $=\left\langle 1 / r_{12}\right\rangle$, the electron kinetic energy $\left\langle p^{2} / 2\right\rangle$, the average electron-nucleus radius $\langle r\rangle$, the electron-pair intracule (relative motion) $\left\langle r_{12}^{n}\right\rangle$ and extracule (center-of-mass motion) $\left\langle R_{12}^{n}\right\rangle$ moments in position space, the corresponding moments $\left\langle p_{12}^{n}\right\rangle$ and $\left\langle P_{12}^{n}\right\rangle$ in momentum space, and some of their parent density functions. All these quantities are functions of one- and two-electron distances either in position or momentum space. In the present paper, we report another aspect of the multiplet states in $p^{2}, p^{3}$, and $p^{4}$ configurations based on the study of the interelectronic angles $\left\langle\theta_{12}\right\rangle$ in position space and $\left\langle\bar{\theta}_{12}\right\rangle$ in momentum space.

For an $N$-electron $(N \geqslant 2)$ system, the average interelectronic angle $\left\langle\theta_{12}\right\rangle$ in position space is defined ${ }^{8}$ by

$$
\left\langle\theta_{12}\right\rangle=\frac{2}{N(N-1)}\left\langle\sum_{i=1}^{N-1} \sum_{j=i+1}^{N} \theta_{i j}\right\rangle,
$$

where the large angle brackets \langle\rangle on the right-hand side stand for the expectation value over the wave function $\Psi\left(\mathbf{x}_{1}, \ldots, \mathbf{x}_{N}\right)$ with $\mathbf{x}_{i}=\left(\mathbf{r}_{i}, s_{i}\right)$ being the combined positionspin coordinates of the electron $i$ and $\theta_{i j}\left(0 \leqslant \theta_{i j} \leqslant \pi\right)$ is the angle spanned by the vectors $\mathbf{r}_{i}$ and $\mathbf{r}_{j}$. The interelectronic angle $\left\langle\bar{\theta}_{12}\right\rangle$ in momentum space is defined ${ }^{9}$ analogously and represents the average angle between any two electron momentum vectors $\mathbf{p}_{i}$ and $\mathbf{p}_{j}$.

For the ${ }^{3} P,{ }^{1} D$, and ${ }^{1} S$ multiplets of five group 14 atoms (C, Si, Ge, Sn, $\mathrm{Pb}$ ) with $p^{2}$ configurations, the ${ }^{4} S,{ }^{2} D$, and ${ }^{2} P$ multiplets of five group 15 atoms $(\mathrm{N}, \mathrm{P}, \mathrm{As}, \mathrm{Sb}, \mathrm{Bi})$ with $p^{3}$ configurations, and the ${ }^{3} P,{ }^{1} D$, and ${ }^{1} S$ multiplets of five group 16 atoms $(\mathrm{O}, \mathrm{S}, \mathrm{Se}, \mathrm{Te}, \mathrm{Po})$ with $p^{4}$ configurations, the average angles $\left\langle\theta_{12}\right\rangle$ and $\left\langle\bar{\theta}_{12}\right\rangle$ were calculated from numerical Hartree-Fock wave functions based on the procedures developed recently in Refs. 8 and 9. Heavy atoms such as $\mathrm{Pb}$ (atomic number $Z=82)$, $\mathrm{Bi}(Z=83)$, and $\mathrm{Po}(Z=84)$ were included in this study to see whether the difference in the angles between different multiplets is common throughout a group of the periodic table.

Table I summarizes the interelectronic angles $\left\langle\theta_{12}\right\rangle$ in position space for the 15 atoms. We first find that in all the cases, $\left\langle\theta_{12}\right\rangle$ are greater than 90 degrees and two electrons are more likely to be on opposite sides of the nucleus than on the same side, though the angle gradually decreases as atomic number $Z$ increases. An important observation in Table $I$ is that a lower multiplet has a larger interelectronic angle $\left\langle\theta_{12}\right\rangle$ than a higher multiplet with no exceptions. It is known in the literature $^{6,10}$ that a lower multiplet has more tight density distribution around the nucleus than a higher multiplet, which results in a smaller $V_{n e}$ and a larger $V_{e e}$ of the lower multiplet. Thus a larger interelectronic angle $\left\langle\theta_{12}\right\rangle$ does not correspond to a smaller interelectronic repulsion energy $V_{e e}$. The results are interpreted that though lower multiplets have larger $V_{e e}$, they still try to reduce $V_{e e}$ by increasing $\left\langle\theta_{12}\right\rangle$. The differences $\Delta\left\langle\theta_{12}\right\rangle$ of the angles relative to the ground multiplet have good negative linear correlations $\Delta\left\langle\theta_{12}\right\rangle$ $\cong a \Delta E+b$ with the corresponding total energy differences $\Delta E$ between the multiplets, though the constants $a(<0)$ and $b(\approx 0)$ differ from one atom to another. As shown in Table I, the correlation coefficients (CCs) are smaller than -0.999 in many cases. The worst is -0.995 for the $\mathrm{C}$ atom. For the group 14 and 16 atoms, the ratio $\Delta E\left({ }^{1} S-{ }^{3} P\right) /$ $\Delta E\left({ }^{1} D-{ }^{3} P\right)$ of the two energy differences is $5 / 2$, while for the group 15 atoms the ratio $\Delta E\left({ }^{2} P-{ }^{4} S\right) / \Delta E\left({ }^{2} D-{ }^{4} S\right)$ is $5 / 3$ in the frozen orbital approximation. ${ }^{7,11-13}$ The HartreeFock ratios were calculated ${ }^{6}$ to be about $2.43,1.65$, and 2.48 for the group 14, 15, and 16 atoms, respectively. In Table I, the corresponding ratios in the angular differences are rather close to these values.

The average interelectronic angles $\left\langle\bar{\theta}_{12}\right\rangle$ in momentum 
TABLE I. Interelectronic angles $\left\langle\theta_{12}\right\rangle$ (in degrees) in position space. CC denotes the correlation coefficient between $\Delta\left\langle\theta_{12}\right\rangle$ and $\Delta E$.

\begin{tabular}{|c|c|c|c|c|c|}
\hline Group 14 atom & ${ }^{3} P$ & ${ }^{1} D$ & ${ }^{1} S$ & $\left({ }^{1} S-{ }^{3} P\right) /\left({ }^{1} D-{ }^{3} P\right)$ & $\mathrm{CC}$ \\
\hline $\mathrm{C}(Z=6)$ & 92.9518 & 92.9210 & 92.8577 & 3.059 & -0.995381 \\
\hline $\mathrm{Si}(Z=14)$ & 91.9367 & 91.9288 & 91.9145 & 2.799 & -0.998145 \\
\hline $\mathrm{Ge}(Z=32)$ & 91.2000 & 91.1984 & 91.1955 & 2.765 & -0.998424 \\
\hline $\operatorname{Sn}(Z=50)$ & 90.8304 & 90.8298 & 90.8287 & 2.792 & -0.998244 \\
\hline $\mathrm{Pb}(Z=82)$ & 90.5717 & 90.5715 & 90.5711 & 2.793 & -0.998258 \\
\hline Group 15 atom & ${ }^{4} S$ & ${ }^{2} D$ & ${ }^{2} P$ & $\left({ }^{2} P-{ }^{4} S\right) /\left({ }^{2} D-{ }^{4} S\right)$ & $\mathrm{CC}$ \\
\hline $\mathrm{N}(Z=7)$ & 93.1726 & 93.1493 & 93.1303 & 1.815 & -0.998003 \\
\hline $\mathrm{P}(Z=15)$ & 91.8900 & 91.8829 & 91.8776 & 1.746 & -0.999302 \\
\hline As $(Z=33)$ & 91.1746 & 91.1730 & 91.1718 & 1.738 & -0.999412 \\
\hline $\mathrm{Sb}(Z=51)$ & 90.8183 & 90.8177 & 90.8173 & 1.744 & -0.999338 \\
\hline $\mathrm{Bi}(Z=83)$ & 90.5660 & 90.5658 & 90.5656 & 1.744 & -0.999343 \\
\hline Group 16 atom & ${ }^{3} P$ & ${ }^{1} D$ & ${ }^{1} S$ & $\left({ }^{1} S-{ }^{3} P\right) /\left({ }^{1} D-{ }^{3} P\right)$ & $\mathrm{CC}$ \\
\hline $\mathrm{O}(Z=8)$ & 93.1548 & 93.1436 & 93.1248 & 2.690 & -0.999329 \\
\hline$S(Z=16)$ & 91.8327 & 91.8292 & 91.8236 & 2.617 & -0.999705 \\
\hline $\operatorname{Se}(Z=34)$ & 91.1469 & 91.1460 & 91.1447 & 2.607 & -0.999745 \\
\hline $\operatorname{Te}(Z=52)$ & 90.8052 & 90.8049 & 90.8044 & 2.617 & -0.999710 \\
\hline Po $(Z=84)$ & 90.5599 & 90.5597 & 90.5595 & 2.617 & -0.999710 \\
\hline
\end{tabular}

space are listed in Table II for the 15 atoms in the groups 14, 15, and 16. As was the case in position space, the angles $\left\langle\bar{\theta}_{12}\right\rangle$ are always larger than 90 degrees and the momentum vectors $\mathbf{p}_{i}$ and $\mathbf{p}_{j}$ of two electrons prefer opposite directions. We find in the table that in every atom, the momentum-space interelectronic angle $\left\langle\bar{\theta}_{12}\right\rangle$ is smaller in a lower multiplet than in a higher multiplet. At present, we do not have any physical interpretation which explains the change in angles $\left\langle\bar{\theta}_{12}\right\rangle$ in relation to the relative stability of the multiplets. When the differences in the angles and total energies relative to the ground multiplet are examined, however, we observe excellent positive linear correlations between them, $\Delta\left\langle\bar{\theta}_{12}\right\rangle$ $\cong \bar{a} \Delta E+\bar{b}$, where the constants $\bar{a}(>0)$ and $\bar{b}(\approx 0)$ differ from an atom to another. As given in Table II, the CCs be- tween $\Delta\left\langle\bar{\theta}_{12}\right\rangle$ and $\Delta E$ are greater than 0.999 in all the cases. In Table II, we also find that the ratios between the two angular differences in momentum space are again close to the constants 5/2 (group 14 and 16 atoms) and 5/3 (group 15 atoms).

The two linear correlations observed above between $\Delta\left\langle\theta_{12}\right\rangle$ and $\Delta E$ and between $\Delta\left\langle\bar{\theta}_{12}\right\rangle$ and $\Delta E$ suggest a direct connection between the angular differences $\Delta\left\langle\theta_{12}\right\rangle$ in position space and $\Delta\left\langle\bar{\theta}_{12}\right\rangle$ in momentum space. Numerical examinations show the anticipation is true; for all the 15 atoms, $\Delta\left\langle\theta_{12}\right\rangle$ and $\Delta\left\langle\bar{\theta}_{12}\right\rangle$ (or $\left\langle\theta_{12}\right\rangle$ and $\left\langle\bar{\theta}_{12}\right\rangle$ ) have negative linear correlations. The CCs range from -0.995 to -1.000 .

In summary, we have shown that for the group 14,15 , and 16 atoms in their ground electronic configurations, a

TABLE II. Interelectronic angles $\left\langle\bar{\theta}_{12}\right\rangle$ (in degrees) in momentum space. CC denotes the correlation coefficient between $\Delta\left\langle\bar{\theta}_{12}\right\rangle$ and $\Delta E$.

\begin{tabular}{|c|c|c|c|c|c|}
\hline Group 14 atom & ${ }^{3} P$ & ${ }^{1} D$ & ${ }^{1} S$ & $\left({ }^{1} S-{ }^{3} P\right) /\left({ }^{1} D-{ }^{3} P\right)$ & $\mathrm{CC}$ \\
\hline $\mathrm{C}(Z=6)$ & 92.6327 & 92.6879 & 92.7661 & 2.416 & 0.999998 \\
\hline $\mathrm{Si}(Z=14)$ & 91.7639 & 91.7727 & 91.7850 & 2.383 & 0.999950 \\
\hline $\mathrm{Ge}(Z=32)$ & 91.1506 & 91.1521 & 91.1542 & 2.351 & 0.999870 \\
\hline $\operatorname{Sn}(Z=50)$ & 90.7950 & 90.7956 & 90.7965 & 2.405 & 0.999981 \\
\hline $\mathrm{Pb}(Z=82)$ & 90.5530 & 90.5533 & 90.5536 & 2.416 & 0.999991 \\
\hline Group 15 atom & ${ }^{4} S$ & ${ }^{2} D$ & ${ }^{2} P$ & $\left({ }^{2} P-{ }^{4} S\right) /\left({ }^{2} D-{ }^{4} S\right)$ & $\mathrm{CC}$ \\
\hline $\mathrm{N}(Z=7)$ & 92.8181 & 92.8660 & 92.8972 & 1.651 & 1.000000 \\
\hline $\mathrm{P}(Z=15)$ & 91.7101 & 91.7198 & 91.7262 & 1.650 & 1.000000 \\
\hline As $(Z=33)$ & 91.1155 & 91.1173 & 91.1185 & 1.647 & 0.999998 \\
\hline $\mathrm{Sb}(Z=51)$ & 90.7780 & 90.7788 & 90.7793 & 1.657 & 0.999998 \\
\hline $\operatorname{Bi}(Z=83)$ & 90.5450 & 90.5453 & 90.5455 & 1.659 & 0.999996 \\
\hline Group 16 atom & ${ }^{3} P$ & ${ }^{1} D$ & ${ }^{1} S$ & $\left({ }^{1} S-{ }^{3} P\right) /\left({ }^{1} D-{ }^{3} P\right)$ & $\mathrm{CC}$ \\
\hline $\mathrm{O}(Z=8)$ & 92.8690 & 92.8886 & 92.9175 & 2.467 & 0.999999 \\
\hline $\mathrm{S}(Z=16)$ & 91.6656 & 91.6704 & 91.6775 & 2.474 & 1.000000 \\
\hline $\operatorname{Se}(Z=34)$ & 91.0846 & 91.0856 & 91.0870 & 2.473 & 0.999999 \\
\hline $\operatorname{Te}(Z=52)$ & 90.7627 & 90.7631 & 90.7638 & 2.486 & 0.999999 \\
\hline Po $(Z=84)$ & 90.5376 & 90.5377 & 90.5379 & 2.490 & 0.999998 \\
\hline
\end{tabular}


lower multiplet of an atom has a larger $\left\langle\theta_{12}\right\rangle$ and a smaller $\left\langle\bar{\theta}_{12}\right\rangle$ than a higher multiplet. The differences in the interelectronic angles $\Delta\left\langle\theta_{12}\right\rangle$ in position space and $\Delta\left\langle\bar{\theta}_{12}\right\rangle$ in momentum space have good linear correlations with the relative stability $\Delta E$ of the multiplets. Linear correlations between $\Delta\left\langle\theta_{12}\right\rangle$ and $\Delta\left\langle\bar{\theta}_{12}\right\rangle$ have also been observed for all the atoms. Preliminary examinations of the ground multiplets of light atoms by multiconfiguration Hartree-Fock calculations show that the inclusion of the electron correlation increases $\left\langle\theta_{12}\right\rangle$ by $1.4,0.9$, and 0.6 degrees, while decreases $\left\langle\bar{\theta}_{12}\right\rangle$ by 1.0, 0.7, and 0.5 degrees, respectively, for the $\mathrm{C}, \mathrm{N}$, and $\mathrm{O}$ atoms. We wish to report whether the present observation at the Hartree-Fock level remain unchanged at the correlated level, when correlated data are available in a systematic manner.
${ }^{1}$ I. N. Levine, Quantum Chemistry, 5th ed. (Prentice Hall, Upper Saddle River, NJ, 2000), p. 328.

${ }^{2}$ R. L. Snow and J. L. Bills, J. Chem. Educ. 51, 585 (1974).

${ }^{3}$ J. Katriel and R. Pauncz, Adv. Quantum Chem. 10, 143 (1977).

${ }^{4}$ R. J. Boyd, Nature (London) 310, 480 (1984).

${ }^{5}$ W. Kutzelnigg and J. D. Morgan III, Z. Phys. D: At., Mol. Clusters 36, 197 (1996).

${ }^{6}$ T. Koga, H. Matsuyama, J. S. Dehesa, and A. J. Thakkar, J. Chem. Phys. 110, 5763 (1999).

${ }^{7}$ T. Koga and H. Matsuyama, J. Chem. Phys. 111, 643 (1999).

${ }^{8}$ T. Koga, J. Chem. Phys. 117, 10493 (2002).

${ }^{9}$ T. Koga, Theor. Chem. Acc. (in press).

${ }^{10}$ T. Koga and Y. Koshida, J. Chem. Phys. 111, 54 (1999).

${ }^{11}$ E. U. Condon and G. H. Shortley, The Theory of Atomic Spectra (Cambridge University Press, Cambridge, 1970).

${ }^{12}$ J. P. Colpa, Mol. Phys. 28, 581 (1974).

${ }^{13}$ J. P. Colpa, A. J. Thakkar, V. H. Smith, Jr., and P. Randle, Mol. Phys. 29, 1861 (1975). 